\title{
Exchange in a Network of Trading Posts
}

\author{
Ross M. Starr ${ }^{1}$ \\ University of California, San Diego \\ Maxwell B. Stinchcombe ${ }^{2}$ \\ University of California, San Diego
}

June 8, 2010

${ }^{1}$ Corresponding author, Department of Economics, University of California - San Diego La Jolla, CA 92093-0508 email: rstarr@ucsd.edu

${ }^{2}$ Department of Economics, University of California, San Diego, 9500 Gilman Drive, La Jolla, CA 92093-0508, email: mstinchcombe@ucsd.edu 


\section{Introduction}

We consider the problem of organizing bilateral trade for given equilibrium prices. In parrticular we wish to establish reasonable sufficent conditions on the structure of transaction costs so that monetary trade is the low cost method of conducting transactions. Walras (1954) suggests that we think of trade taking place at a family of trading posts, one for each pair of goods.

In order to fix our ideas, we shall imagine that the place which serves as a market for the exchange of all the commodities $(A),(B),(C),(D) \ldots$ for one another is divided into as many sectors as there are pairs of commodities exchanged. We should then have $\frac{m(m-1)}{2}$ special markets each identified by a signboard indicating the names of the two commodities exchanged there as well as their prices or rates of exchange ...

Thus for each good $i$ and each good $j$, there may be a trading post for the trade of $i$ and $j$ for one another. This leads to an array, as Walras notes, of approximately $\frac{1}{2} n^{2}$ trading posts where $n$ is the number of commodities. In most economies we see far fewer pairs of goods in active trade. Most trade takes place between the $\mathrm{n}$ goods and a single good differentiated as money. The number of moneys is small, typically unity, in a single economy (though this raises the tricky question of what are the boundaries of an economy). A monetary trade structure reduces the number of active trading posts from approximately $\frac{1}{2} n^{2}$ to approximately $n$. Is this reduction the distinguishing feature of a monetary economy? What motivates the collapse of the full array of trading posts? Professor Tobin (1980) suggests that the use of a single money is in the nature of a public good, perhaps reflecting a scale economy at the level of the economy as a whole.

Social institutions like money are public goods. Models of ... competitive markets and indiviual optimizing agents ... are not well adapted to to exxplaining the existence and quantity of public goods. ... Both [money and language] are means of communication. The use of a particular language or a particular money by one individual increases its value to other actual or potential users. Increasing returns to scale, in this sense, limits the number of languages or moneys in a society and indeed explains the tendency for one basic language or money to monopolize the field.

In this article we will formalize the view expressed above (without implicating Tobin) by characterizing the use of a ull-ique money as the result of an optimizing 
(cost miniimizing) decision on the array of active trading posts in the model posited by Walras. In particular, we will develop sufficient conditions to allow us to derive the self-confirming public good character of the choice of the monetary instrument.

In this article we will formalize the view expressed above (without implicating Tobin) by characterizing the use of a unique money as the result of an optimizing (cost miniimizing) decision on the array of active trading posts in the model posited by Walras. In particular, we will develop sufficient conditions to allow us to derive the self-confirming public good character of the choice of the monetary instrument.

The character of the cost structure in this model is formally very similar to the cost structure in Hendricks, Piccione, and Tan (1992) and Starr and Stinchcombe (1992). In Starr and Stinchcombe (1992) we analyzed cost minimizing structure of airline route systems. There are $n$ cities to be linked by airline routes. Direct flights between each city pair implies $\frac{1}{2} n(n-1)$ air routes. A hub-and-spoke network uses only $n-1$, however at the cost of causing some passengers to travel redundant mileage and to incur the delays of changes of plane. The saving in routes is efficient if there are strong set-up costs on each route and if the costs of extra mileage are correspondingly low. A transportation cost function displaying sufficient scale economy causes the route structure of the airlines to minimize costs for a given level of service with a hub and spoke network. All travel there passes through the hub to take advantage of scale economies (declining average cost per passenger) at the level of a single flight.

In the present study of trading structure, we will find similarly that for suitable transsaction cost structures, transaction cost minimization implies that all trade pass through a unique (but arbitrary) money to take advantage of scale economies (declining average cost per unit transaction) at the level of the trading post. If we depict the structure of trading relations as a graph, direct trade of each good for each other good would look like the map of an airline route system with direct links among all $n$ cities ( $n$ commodiities). The graph of a monetary trade system will look like a hub and spoke system with the monetary commodity at the hub. This observation gave rise to an exam question at UCSD: "Why is money like Chicago's O'Hare airport?"

Most models of money as a medium of exchange, following Jevons (1875), (see Kiyotaki and Wright (1989), Ostroy and Starr (1974), and the models discussed in Ostroy and Starr(1990)) focus on trade as an interaction between individual agents in the economy - the primitive unit is the pair of traders. The present study, following Walras' discussion above, treats the primitive unit as pairs of commodities in which active trade takes place. In this it follows the approach of Rogawski and Shubik (1986), and of Shubik (1973, 1993). 
The pairs of goods in active trade for one another will be described by a binary relation on the set of goods. A trading relation in which most goods are traded for one another will be nonmonetary; a trading relation in which there is a distiguished good for which most goods are traded but where most goods are not traded directly for one another is monetary with the distinguished good acting as money. The trading relation will be determined endogenously as a cost minimizer subject to fulfillment of demands and supplies. We seek to establish the observation that "money buys goods and goods buy money but goods do not buy goods" Clower (1965), as the result of optimization rather than as an assumption.

\section{Initial Conditions}

Let there be $n$ goods, denoted $i=1,2,3, \ldots, n$, constituting the set $N$. Prices will be denoted by $p \in P$, the unit simplex in $\mathbb{R}_{+}^{n}$. Let the typical trading household be denoted $h$, an element of the (finite) set of households $\mathcal{H}$, $\# \mathcal{H} \geq 2$. For each $h \in \mathcal{H}$, $z^{h} \in \mathbb{R}^{n}$ is $h$ 's excess demand vector. We assume that $z^{h}$ fulfills household $h$ 's budget constraint (1) and that market excess demands at $p$ clear (2). Hence we assume:

$$
\begin{gathered}
p \cdot z^{h}=0, \text { and } \\
\sum_{h \in \mathcal{H}} z^{h}=0 .
\end{gathered}
$$

Let $\tilde{Z} \equiv\left[z^{h}\right]_{h \in \mathcal{H}}$, where $[\cdot]_{h \in \mathcal{H}}$ denotes the $\# \mathcal{H} \times n$ matrix. We maintain the following non-triviality assumptions on the problem of organizing trade:

All goods are traded, that is, $\widetilde{Z}$ has no columm of $O$ 's, and $\# \mathcal{H}+n<\# \mathcal{H} \cdot n$.

We describe an array of trading posts as a relation $R$ on $N$ following Haller (1989a, 1989b). The intended interpretation is that $i R j$ if there is a trading post for trade of $i$ and $j$. The notation $\neg i R i$ denotes "it is not the case that $i R j . "{ }^{1} \mathrm{By}$ convention, there is no trading post for trade of $i$ with $i$, that is, $\neg i R i$ for all $i \in N$. Viewing $R$ as a subset of $N \times N$, this can be rewritten as $\Delta \frown R=\theta$, where $\Delta$ is the diagonal in $N \times N$. When convenient, we shall also regard any relation $S$ on $N$ as a correspondence by defining $S(i)=\{j: i R j\}$. Thus, for a trading post structure $R, R(i)$ is the set of $j$ that can be acquired with $i$ by direct trade.

For arbitrary relations $\mathrm{S}$ on $\mathrm{N}$ we will use the following notation:

\footnotetext{
${ }^{1}$ Stinchcombe (1968) also contains a variety of network models of social phenomena.
} 
1. For $m \in N$, define the relation $S^{m}$ by $i S^{m} j$ if there are $m$ commodities $i=$ $i 1, i_{2}, \ldots, i_{m-1}, i_{m}=j$, such that $i_{j} R i_{j+1}$ for $j=1,2, \ldots, m-1$. For a trading post structure $R, R^{m}(i)$ is the set of goods $j$ that can be acqired by trade starting with $i$ in exactly $m$ successive trades.

2. For $m \in N$, let $\bar{S}^{m}$ denote $\smile_{m^{\prime} \leq m} S^{m^{\prime}}$. For a trading post structure $R, i \bar{R}^{m} j$ if it is possible to trade $i$ for $j$ in $m$ or fewer successive trades. $\bar{S}^{n}=\bar{S}$ is the transitive closure of $S$.

3. For $i \in N, S_{-}(i)$ denotes the set $\{j \in N: j S i\}$. For a trading post structure $R, R_{-}(i)$ is the set of goods for which $i$ can be acquired by a single direct trade, $\bar{R}_{-}^{m}(i)$ is the set of goods for which $i$ can be acquired in $m$ or fewer trades, and $R_{-}^{m}(i)$ denotes the the set of goods for which $i$ can be aquired in exactly $m$ trades.

We will restrict attention to trading post structures $R$, connected in the sense that it is possible to trade each good $i$ for any other $j$ in a succession of trades using the trading posts available. Formally, this is the requirement that

$$
\bar{R}^{n}=N \times N
$$

or equivalently, $E \subset \bar{R}^{n}$, where $E=\{N \times N\} \backslash \Delta$ is the relation $i E j$ for all $i \neq j$.

Finally we require

$$
R \text { is a symmetric binary relation on } N \times N \text {. }
$$

That is, we suppose that the trading post where $i$ can be traded for $j$ is the trading post where $j$ can be traded for $i$.

As an aid to intuition, it is often convenient to have a matrix representation of trading post structures. For this purpose we use adjacency matrices (Lawler (1976, p. 22)). For example, if $R$ is the cyclical trading post structure $1 \rightarrow 2,2 \rightarrow 3,3 \rightarrow 4$, and $4 \rightarrow 1$, then the following four matrices represent $R, R^{2}, R^{3}, \bar{R}^{3}$, and $R^{4}$.

$R=$\begin{tabular}{|c|c|c|c|c|}
\hline$i \backslash j$ & 1 & 2 & 3 & 4 \\
\hline 1 & & $\cdot$ & & \\
\hline 2 & & & $\cdot$ & \\
\hline 3 & & & & $\cdot$ \\
\hline 4 & $\cdot$ & & & \\
\hline
\end{tabular},$R^{2}=$\begin{tabular}{|c|c|c|c|c|c|}
\hline$i \backslash j$ & 1 & 2 & 3 & 4 \\
\hline 1 & & & $\cdot$ & \\
\hline 2 & & & & $\cdot$ \\
\hline 3 & $\cdot$ & & & \\
\hline 4 & & $\cdot$ & & \\
\hline
\end{tabular},$R^{2}=$\begin{tabular}{|c|c|c|c|c|c|}
\hline$i \backslash j$ & 1 & 2 & 3 & 4 \\
\hline 1 & & & & $\cdot$ \\
\hline 2 & $\cdot$ & & & \\
\hline 3 & & $\cdot$ & & \\
\hline 4 & & & $\cdot$ & \\
\hline
\end{tabular}




$\bar{R}^{3}=$\begin{tabular}{|c|c|c|c|c|}
\hline$i \backslash j$ & 1 & 2 & 3 & 4 \\
\hline 1 & & $\cdot$ & $\cdot$ & $\cdot$ \\
\hline 2 & $\cdot$ & & $\cdot$ & $\cdot$ \\
\hline 3 & $\cdot$ & $\cdot$ & & $\cdot$ \\
\hline 4 & $\cdot$ & $\cdot$ & $\cdot$ & \\
\hline
\end{tabular}, and finally, $\bar{R}^{4}=$\begin{tabular}{|c|c|c|c|c|c|}
\hline$i \backslash j$ & 1 & 2 & 3 & 4 \\
\hline 1 & $\cdot$ & $\cdot$ & $\cdot$ & $\cdot$ \\
\hline 2 & $\cdot$ & $\cdot$ & $\cdot$ & $\cdot$ \\
\hline 3 & $\cdot$ & $\cdot$ & $\cdot$ & $\cdot$ \\
\hline 4 & $\cdot$ & $\cdot$ & $\cdot$ & $\cdot$ \\
\hline
\end{tabular}

In this case, $\bar{R}^{3}$ is equal to $E$, the trading post structure where every ood is connected by a direct trade to every other good.

In a smilar fashion, if $R$ is the monetary trading post structure with the money $k=1$, the corresonding graphs are:

$R=$\begin{tabular}{|c|c|c|c|c|}
\hline$i / j$ & 1 & 2 & 3 & 4 \\
\hline 1 & & $\cdot$ & $\cdot$ & $\cdot$ \\
\hline 2 & $\cdot$ & & & \\
\hline 3 & $\cdot$ & & & \\
\hline 4 & $\cdot$ & & & \\
\hline
\end{tabular} , and $R^{2}=$\begin{tabular}{|c|c|c|c|c|c|}
\hline$i / j$ & 1 & 2 & 3 & 4 \\
\hline 1 & $\cdot$ & $\cdot$ & $\cdot$ & $\cdot$ \\
\hline 2 & $\cdot$ & $\cdot$ & $\cdot$ & $\cdot$ \\
\hline 3 & $\cdot$ & $\cdot$ & $\cdot$ & $\cdot$ \\
\hline 4 & $\cdot$ & $\cdot$ & $\cdot$ & $\cdot$ \\
\hline
\end{tabular}

Definition 1 A trading post structure $R$ is connected if it is possible to trade from each good $i$ to any other good $j$ using the trades available in the trading post structure. Formally, $R$ is connected if $\bar{R}^{n}=N \times N$ or, equivalently, $E \subset \bar{R}^{n}$.

We seek now to describe the array of active trading posts as an optimizing decision.

\section{Trade Plans}

Household $h$ 's trading plan is characterized by the $n \times n$ matrix $V^{h}=\left(v_{i j}^{h}\right)$. The intended interpretation is that $v_{i j}^{h}$ is $h$ 's net receipt of $i$ in exchange for $j: v_{i j}^{h}<0$ is a delivery of $i$ in exchange for $j ; v_{i j}^{h}>0$ is a receipt of $i$ in return for $j$. We expect each good traded to be paid for at the trading post by opposite delivery of the other good traded at the post.

$$
p_{i} v_{i j}^{h}=-p_{j} v_{j i}^{h} .
$$

Further, the trading plans should seek fully to implement $h$ 's demands $z^{h}$.

$$
\sum_{j} v_{i j}^{h}=z_{i}^{h} .
$$


However, $h$ 's trading plans should be consistent with the array of trading posts in active operation according to the relation $R$. Thus

$$
v_{i j}^{h} \neq 0 \text { only if } i R j \text { or } j R i \text {. }
$$

It is convenient to be able to distinguish $h$ 's planned receipts $\left(v_{i j}^{h}>0\right)$ from his planned deliveries $\left(v_{i j}^{h}<0\right)$. To this end, let

$$
v_{i j}^{h+}=\left\{\begin{array}{c}
v_{i j}^{h} \text { if } v_{i j}^{h} \geq 0 \\
0 \text { if } v_{i j}^{h}<0
\end{array},\right.
$$

and let

$$
v_{i j}^{h-}=\left\{\begin{array}{c}
0 \text { if } v_{i j}^{h} \geq 0 \\
-v_{i j}^{h} \text { if } v_{i j}^{h}<0
\end{array}\right.
$$

so that $h$ 's planned receipts are $v_{i j}^{h+}$, planned deliveries are $v_{i j}^{h-}$, and $v t=v_{i j}^{h}=$ $v_{i j}^{h+}-v_{i j}^{h-}$. We discuss the endogenous determination of the $v_{i j}^{h}$ below.

To illustrate how $R$, the array of active trading posts, affects the pattern of trade, consider three very different cases:

- $E$ (the universal array) where there are $\frac{1}{2} n(n-1)$ trading posts and all goods can trade against each other,

- A linear array with $n-1$ trading posts and only prescribed pairs of goods trade against one another, each good trading against two other goods only, $i_{1} \leftrightarrow i_{2} \leftrightarrow \ldots i_{n}$, and

- Monetary exchange, where there are $n-1$ trading posts only but a single good, "money," is one of the goods traded in each pair.

When $E$ is the trading array, all goods trade directly for one another. There are $\frac{1}{2} n(n-1)$ trading posts in use. Each agent goes to the trading posts dealing in the combination of goods $i j$ where $i$ is one of his excess demands and $j$ is one of his excess supplies. His dollar (unit of account) volume of trade is precisely equal to the value of his excess supplies plus the value of his excess demands, that is, each excess supply is traded once for an excess demand.

In the linear array, there are $n-1$ trading posts in use. There will typically be only two trading posts available where $j$, of which the trader has an excess supply, is traded. She chooses the one that will lead eventually to a post trading $i$ for which she has an excess demand. The trader goes to the first chosen trading post to deliver her excess supply and withdraw an equal value of the other good traded at the post. 
She retrades this good at the adjacent post for a third good, and then to an adjacent post for a fourth good, ... , eventually arriving with a suitable supply at a post dealing in her desired excess demand for which she then trades. Trading volumes in this setting may be many times the value of excess demands, since each good may be retraded several times as a carrier of value between trading posts.

In the monetary array, there are $n-1$ trading posts in use. The trader goes to each trading post dealing in a (non-money) good for which he has an excess supply and exchanges it for the money good. He then goes to the trading post where one of his demanded goods is traded and exchanges the money for the demanded good. The volume of trade is approximately two times the value of excess supplies plus excess demands (since each of these is traded once for an equal value of money). The number of active trading posts is the same as in the linear array, but the volume of trade is much smaller. The number of trading posts is $\frac{2}{n}$ ths the number in the universal array and the gross volume of trade is twice as high.

There are a few other distinctions of the monetary trading array to note. The classical explanation for the use of money is the absence of a double coincidence of wants, Jevons (1875). That is, absence of a condition where for each agent with an excess supply of $i$ and an excess demand for $j$, there is a corresponding agent with the opposite demands and supplies. In the present model, the rationale for monetary trade does not depend on the absence of a double coincidence of wants. It is rather a scale economy at the level of the trading arrangements of the economy as a whole. If households' demands and supplies are sufficiently diverse (so that nonmonetary trade would use a large variety of trading posts even with double coincidence) then monetary trade is the low cost trading arrangement when the fixed cost of a trading post is high and marginal costs of trade are low, even in the (rare) case of double coincidence of wants.

Nonmonetary trade in the trading post model, even ignoring transaction costs, has a distinct problem that monetary trade can solve, market clearing at the trading post. Though markets clear for all goods (2), there is no guarantee that the flows at any single trading post will clear, i.e. that $\sum_{h \in \mathcal{H}} v_{i j}^{h}=0$. This implies that there is an untreated issue of interpost trade in the nonmonetary model. However, monetary trade resolves this issue. All trade for good $i$ in a monetary trading array is at the trading post for $i$ and money. Hence in monetary trade (1) implies purchases equal sales at the trading post. This follows simply because monetary trade is exchange of excess demands and supplies for money and (1) guarantees that the excess demands and supplies clear. 


\section{The Central Problem of Efficient Exchange}

How should an economy arrange its bilateral trade? Some arrays of trading posts may be more costly than others to arrange, sustain, and use for trade.

In particular, if a choice of $R$ (like the linear array) meant that most trades had to go through a circuitous trading sequence, or an alternative choice (like $E$ ) meant that many redundant trading posts were kept open, then these might represent excessively costly, nonoptimal choices of trading array. Is it efficient or desirable to organize trade as monetary trade? Given $p \in P$, $z^{h}$ fullfilling (1), (2), then an optimal choice of $R$ would minimize the cost of $V, R$ subject to (5) - (7).

For all $i, j \in N$, pairs with $i \neq j$, let $\alpha_{i j}$ be a fixed cost of running the trading post for the pair of goods $i j$. Because we assume that the trading post where $i$ is traded for $j$ is the same as the trading post where $j$ is traded for $i$, it is natural to assume that $\alpha_{i j}=\alpha_{j i}$. For $i \in N$, let $\beta_{i}$ be the marginal cost of flows of good $i$. Let $\widetilde{\alpha}$ and $\widetilde{\beta}$ and be the vectors of $\alpha$ 's and $\beta$ 's. We study cost functions of the form

$$
C(R, \widetilde{Z} ; \widetilde{\alpha}, \widetilde{\beta})=\sum_{\substack{i>j \\ i R j}} \alpha_{i j}+\sum_{i>j} \beta_{i}\left(m_{i j}^{+}(R, \widetilde{Z}, \widetilde{\beta})+m_{i j}^{-}(R, \widetilde{Z}, \widetilde{\beta})\right)
$$

where $m_{i j}^{+}(R, \widetilde{Z}, \widetilde{\beta})$ denotes quantity of $i$ delivered to households from the market with $j$, and $m_{i j}^{-}(R, \widetilde{Z}, \widetilde{\beta})$ denotes quantity of $i$ sent by households to the market with $j$. That is, $m_{i j}^{+}(R, \widetilde{Z}, \widetilde{\beta})=\sum_{h \in \mathcal{H}} v_{i j}^{h+}$ and $m_{i j}^{-}(R, \widetilde{Z}, \widetilde{\beta})=\sum_{h \in \mathcal{H}} v_{i j}^{h-}$ where households choose their planned trades, $V^{h}$, in response to $R, \widetilde{Z}$, and (potentially) to $\widetilde{\beta}$. Specifically, we assume that household $h$ chooses their trading pattern, $V^{h}$ to be consistent with (5) - (7), and to minimize

$$
\sum_{i>j} \beta_{i}^{h}\left(v_{i j}^{h+}+v_{i j}^{h-}\right)
$$

where each $\beta_{i}^{h}>0$. A final piece of notation: At various points in the analysis it will be convenient to talk about phenomena that are true for "small" vectors $\mathbf{x} \in \mathbb{R}^{\ell}, \ell \in \mathbb{N}$. To this end, we will use the notation " $\mathbf{x} \simeq \mathbf{0}$ " which is read as " $\mathbf{x}$ is small," or " $\mathbf{x}$ is negligible." Thus, the statement "if $\mathbf{x} \simeq \mathbf{0}$ then $S(\mathbf{x})$ is true" means "if $\mathbf{x}^{n}$ is a sequence with $\left\|\mathbf{x}^{n}\right\| \rightarrow 0$, then for all sufficiently large $n, S\left(\mathbf{x}^{n}\right)$ is true," or equivalently, "there is a neighborhood of $\mathbf{0}$ in which $S(\mathbf{x})$ is true." Thus, negligible vectors or numbers represent the behavior of small numbers and vectors where the exact size of "small" can vary by context. Note that the finite sum of negligible vectors or numbers is again negligible. The notation " $\mathbf{y} \simeq \mathbf{x}$ " is short-hand for " $\mathrm{y}-\mathrm{x} \simeq 0$." 


\section{$5 \quad$ Small Fixed Costs}

In this section we consider cost functions for which the marginal costs of trade flows dominate, and the fixed costs are negligible. Section 6 considers the opposite case of negligible marginal costs.

Here, total costs are virtually linear in the quantity of goods traded. The cost miniimizing trading post structure is, for generic $\widetilde{Z}, E(\widetilde{Z})$, the array of trading posts containing all pairs of goods which at least one household would like to trade,

$$
E(\widetilde{Z})=\left\{(i, j): \text { for some } h \in \mathcal{H}, \operatorname{sgn}\left(z_{i}^{h}\right) \neq \operatorname{sgn}\left(z_{j}^{h}\right)\right\}
$$

In other words, $E(\widetilde{Z})$ is the universal relation $E$ minus all those pairs of goods that no household mighty want to trade.

Genericity enters as follows. Suppose that it were possible to partition $N$ into two disjoint sets of goods, $N_{1}$ and $N_{2}$, with the property that

$$
\text { for all } h \in \mathcal{H}, \sum_{i \in N_{1}} p_{i} z_{i}^{h}=0 \text { and } h \in \mathcal{H} \sum_{i \in N_{2}} p_{i} z_{i}^{h}=0 .
$$

In this case, there would be no need to have any trading links between the sets $N_{1}$ and $N_{2}$ because the households could separately fulfill their desired net trades within the $N_{1}$ goods and within the $N_{2}$ goods. For any given vector of prices $p$, it is a generic property of $\widetilde{Z}$ that no such partition of $N$ is possible, and it is this genericity that we use. In particular, it implies that any trading structure that allows the households to complete all of their desired net trades must be connected. This genericity also implies that if a household might want to trade goods $i$ and $j$, then they must in fact trade them in order to exactly fulfill their desired net trades.

The trading post array $E(\widetilde{Z})$ is the solution in this case because with any other system, some trader(s) would be obliged to trade indirectly, increasing the volume of trade flows and hence increasing costs by a non-negligible amount. In particular, a system of monetary trade with all flows through a single good $k$ cannot be optimal, except in the extreme (and non-generic) case that all households either have an excess supply of only one good and that single good is $k$, or they have excess demand for only one good and that single good is $k$.

We wish to describe the efficient trading post arrays, $R$, as cost minimizing solutions for the following problem,

$$
[\operatorname{Symmetric}(m)] \min _{R} C(R, \widetilde{Z}) \text { s.t. } E \subseteq \bar{R}^{m} \text { and } R \text { symmetric. }
$$

Here $m$ is a positive integer indicating the maximum number of trading posts at which the household may need to trade successively in order to exchange good $i$ for 
good $j$. In other words, the constraints are that $R$ be symmetric and connected in $m$ or fewer steps. Note that when $m \geq n, \bar{R}^{m}=\bar{R}^{n}=\bar{R}$, so that the problem Symmetric $(n)$ is has no restrictions beyond symmetry and connectedness. We will use the restriction implied by $m$ only once in Theorem 2 of Section 6 . There we intend it as a proxy for the costs of many successive trades. In this section, the cost of many succesive trades is the object of analysis.

Theorem 2 If $\widetilde{\alpha}>\mathbf{0}$ and $\widetilde{\alpha} \simeq \mathbf{0}$ and $\widetilde{\beta}>\mathbf{0}$, then for generic $\widetilde{Z}, E(\widetilde{Z})$ is the umque solution to the problem Symmetric $(n)$.

Proof: We present the proof in two parts. First we argue at the level of the household that direct trade is optimal. Second, we show that the same result holds at the level of the economy. The essential intuition in both cases is that any pattern of trade other than the most direct increases indirect flows and this increases cost.

Consider first household $h$ 's decision to choose the trading pattern $v_{i j}^{h}$. By assumption, they are trying to minimize a function which can be rewritten as $\frac{1}{2} \sum_{i, j}\left(\beta_{i}^{k}+\right.$ $\left.\beta_{j}^{h}\right) \cdot\left(v_{i j}^{h+}+v_{i j}^{h-}\right)$. Suppose that it is possible to re-route a quantity of trade $\delta$ between goods 1 and 2 through good 3 . This leads to a change of $\frac{1}{2}$ times $\left(\beta_{1}^{h}+\beta_{2}^{h}\right) \delta$ - $\left(\beta_{1}^{h}+\beta_{3}^{h}\right) \delta+\left(\beta_{2}^{h}+\beta_{3}^{h}\right) \delta=\beta_{2}^{h} \delta$ in the objective function. Thus, for any proposed route structure, moving in a direction of more direct trade, $\delta<0$, lowers the function to be minimized. Thus, household $h$ will choose direct trade if possible.

Turning now to the economy-wide problem, substituting $\beta_{i}$ and $\beta_{j}$ for $\beta_{i}^{h}$ and $\beta_{j}^{h}$ in the above argument shows that the most direct possible trade also solves the problem of minimizing the total cost of flows. Because $\widetilde{\alpha} \simeq \mathbf{0}$, the cost of any given trading post that some household might want to is negligible in comparison to the saving to be had by including it in the trading post array. Finally, because $\widetilde{\alpha}>\mathbf{0}$, we save by omitting unused trading posts, and the result is $E(\widetilde{Z})$.

The following demonstrates some of the range of trading post arrays that may arise as solutions in Theorem 1.

Example 1: Suppose that the matix of excess demands is given by

\begin{tabular}{|c|c|c|c|c|}
\hline$h \backslash i$ & 1 & 2 & 3 & 4 \\
\hline 1 & +1 & -1 & 0 & 0 \\
\hline 2 & 0 & +1 & -1 & 0 \\
\hline 3 & 0 & 0 & +1 & -1 \\
\hline 4 & -1 & 0 & 0 & +1 \\
\hline
\end{tabular}


so that $E(\widetilde{Z})$ is the symmetric, cyclical trading post structure satisfying $1 R 2,2 R 3$, $3 R 4$, and $4 R 1$.

\section{Small Marginal Costs and Monetary Trade}

We now examine the implications of negligible marginal costs, a situation where the fixed costs of trading posts dominate, formally, $\widetilde{\beta} \simeq \mathbf{0}$ and $\widetilde{\alpha}>\mathbf{0}$. In this case, the minimal total cost is achieved by establishing the minimal number of trading posts consistent with $E \subset \bar{R}^{m}$, that is, connecting all commodities with the minimal number of trading posts. This is $(n-1)$. The trading posts could then be in the form of a line, $i_{1} \leftrightarrow i_{2} \leftrightarrow \ldots \leftrightarrow i_{n}$, a hub and spoke array (monetary trade), or a variety of alternative configurations.

Line structures of trading post arrays have an obvious drawback from the point of view of the traders; a single exchange of one good for another in a line array might involve as many as $n-1$ distinct transactions as an excess supply was traded for each of a succession of intervening goods with active trading posts, eventually achieving the good in excess demand. In a cycle this trade might require $\frac{1}{2}(n-1)$ transactions. Our proxy for this cost is the restriction that traders need make no more than $\mathrm{m}$ distinct transactions in order to complete a two good (one supply, one demand) trade. If $m=1$, then the only connected trading post structure is a system of trading posts for every commodity pair, $E$. The case $m=2$ corresponds to retrading more than once being prohibitively expensive. In this context, with low variable costs and fixed costs approximately uniform, we are led to monetary trade networks with a single money, Theorem 2. Example 2 shows that the assumption of approximately uniform fixed costs cannot be loosened and gives some sense of the range of possible cost minimizing trading arrays.

By contrast, when the fixed costs are not uniform and $\mathrm{m}$ is unrestricted, we are led to multi-money systems, Theorem 3. In Example 3 below, multiple currencies

( $\$$ and $£)$ arise as efficient allocations where the cost structure favors concentrating trade in US goods on $\$$ and UK goods on $£$ allowing trade between US and UK goods to require three steps. Here, $m=3$ corresponds to US goods to $\$, \$$ to $£, £$ to UK goods, rather than the $m=2$ of a monetary trading array with a single money.

It seems then that the emergence of a single money is unlikely. However, Corollary 4 gives sufficient conditions for a good to be a "natural" money under the conditions of Theorem 3. This leads us to a brief discussion of the rate of return dominance puzzle and its relation to this work. 


\subsection{The Emergence of a Single Money}

We begin with our definition of a money.

Definition 3 A monetary trade system with a single money $k \in N$ is a relation $R$ such that $i R k$ and $k R i$ for all $i \neq k$, and . $\neg i R j$ if neither $i$ nor $j$ is equal to $k$.

Note that monetary trade systems with a single money are connected, indeed, R? $=\mathrm{N} \times \mathrm{N}$. Further, they have $(\mathrm{n}-1)$ trading posts in them.

Theorem 4 If $\# N \geq 3, \widetilde{\beta} \simeq \mathbf{0}, \widetilde{\alpha} \simeq \alpha \widetilde{\mathbf{1}}, \alpha>0$, then the only solutions to Symmetric(2) are monetary trade systems with a single money.

Proof: Because $\widetilde{\beta} \simeq \mathbf{0}$ and $\widetilde{\alpha} \simeq \alpha \widetilde{\mathbf{1}}$, it is sufficient to show that monetary trade systems with one money are the only solutions to the problem

$$
\min _{R} \# R \text { s.t. } E \subset \bar{R}^{2}, R \text { symmetric. }
$$

Monetary trade systems with a single money satisfy the constraint and have (n 1 ) trading posts in them. Hence, if $R$ is a solution to (15), then $\# R \leq 2(n-1)$.

Let $\underline{m}$ denote $\min _{i \in N} \# R(i)$. Because $R$ is connected, we know that $\underline{m}>0$. If $\underline{m} \geq 2$, then $\# R \geq 2 n$, a contradiction. Hence $\underline{m}=1$. Let $m=\max _{i \in N} \# R(i)$. As an intermediate step in the proof, we will show that if $n=\# N \geq 4$, then $\bar{m}=n-1$.

Let $i^{\prime}$ be a point in $N$ such that $\# R\left(i^{\prime}\right)=1$. If $\bar{m} \leq n-3$, then it is impossible to get from $i^{\prime}$ to some point $j \in N$ in 2 or fewer trades, violating the constraint. Hence, either $\bar{m}=n-2$ or $\bar{m}=n-1$.

Suppose that $\bar{m}=n-2$ and let $k$ be a point (commodity) in $N$ with $\# R(k)=$ $n-2$. Because $\# R \leq 2(n-1)$ and $\underline{m}=1$, there is at most 1 point $j \in N \backslash\{k\}$ such that $\# R(j)=2$. Because $N$ has at least 4 points in it, $\# R\left(i^{\prime}\right)=\# R\left(i^{\prime \prime}\right)=1$ for some pair $i^{\prime} \neq i^{\prime \prime}$ in $N$. But because $\bar{m}=n-2$, from either $i^{\prime}$ or $i^{\prime \prime}$ (or both), it is impossible to reach some point $j \in N$. This violates the constraint, hence $\bar{m}=n-1$.

To finish the proof, let $k$ be that point in $N$ such that $\# R(k)=n-1$ (there clearly cannot be two $k$ with this property). Because $\bar{m}=n-1, \underline{m}=1$, and $\# R \leq 2(n-1)$, we know that for all $i \neq k, \# R(i)=1$. If for some $i \neq k, R(i) \neq\{k\}$, then there is some $j \in N$ that cannot be reached from $i$ in two steps, violating the constraint. Hence $S$ is a monetary trade system with money $k$.

If $\widetilde{\alpha} \nsim \alpha \widetilde{\mathbf{1}}$, the conclusions of Theorem 2 can fail quite conclusively.

Example 2 (Yes we have no money): Let $\# N=4, \widetilde{\beta}, \widetilde{\gamma} \simeq \mathbf{0}$. Suppose that $\alpha_{i j}=\alpha_{j i}, \alpha_{12}=\alpha_{23}=\alpha_{34}=\alpha_{41}=1$ while $\alpha_{13}=\alpha_{24}=x$. Let $S$ denote a monetary 
trade system with money being any point in $N$. The cost of $S$ is $4+2 x$ plus a finite sum of negligibles. Let $R$ denote the symmetric trading post structure connecting all pairs except $\{1,3\}$ and $\{2,4\}$. The cost of $R$ is 8 plus a finite sum of negligibles. It is easy to check that $\bar{R}^{2}=E$. For $x>2, R$ is strictly better than $S$.

\subsection{The Emergence of Multiple Moneys}

To state our next result, it is convenient to use some concepts from graph theory.

Definition 5 a graph $G$ on $N$ is a pair $(N, A)$ where $N$ is a finite set and $A$ is a collection of unordered pairs, arcs, of elements of $N$. A path from $i$ to $j$ in a graph $G$ is an ordered sequence of nodes in $N, i=i_{1}, i_{2}, \ldots, i_{K-1}, i_{K}=j$, where each arc $\left(i_{k}, i_{k+l}\right), k=1, \ldots, K-1$ is in $A$. $G$ is connected if there is a path from each $i$ to each $j$ in $N$. For $h \geq 2$, an $h$-cycle is a path from $i$ to $i$ containing $h$ arcs in which no node except $i$ is repeated. If $G$ is connected and contains no $h$-cycles, $h \geq 3$, it is called a tree.

We relate this to our trading post arrays by

Definition 6 For a symmetric binary relation $R$, the graph associated with $R$ is $(N, A)$ where $A$ is the set of unordered pairs $(i, j)$ such that $i R j$.

Note that $\# R=2 \cdot \# A$ when $(N, A)$ is the graph associated with $R$.

Unlike Theorem 2, the following result puts no restriction on the number of trading posts a household may need to visit in order to fulfill their desired net trades.

Theorem $\mathbf{7}$ If $\widetilde{\beta} \simeq \mathbf{0}$ and $\widetilde{\alpha}>\mathbf{0}$, then the graph associated with any solution, $R$, to Symmetric(n) is a tree. In particular, $\# R=2(n-1)$.

Proof: Let $R$ be a solution to $\operatorname{Symmetric}(n)$ and let $G$ be the graph associated with $R$. If $R$ has an $h$-cycle, $h \geq 3$, removing one of the arcs reduces cost by a nonnegligible amount because $\widetilde{\alpha}>\mathbf{0}$, increases costs by a negligible amount because $\widetilde{\beta} \geq \mathbf{0}$, and it leaves $R$ connected. Therefore, $R$ has no $h$-cycles, $h \geq 3$ and is a tree. But this is equivalent to $G$ being connected and having $n-1$ arcs (Lawler (1976, Prop. 5.2)).

The monetary trade systems that arise in Theorem 2 are trees where only one good, the money, is directly connected to many other commodities. The following two-money example indicates some of the range of tree structures that may arise as solutions under the conditions of Theorem 3. 
Example 8 (An International Perspective) Suppose that $n=n_{1}+n_{2}$, and that $n_{1}$ of the commodities in $N$ are in the United States and $n_{2}$ in the United Kingdom. Pick a distinguished node in the U.S. and call it \$US, pick a distinguished node in the U.K. and call it £Sterling. Consider the symmetric relation connecting all of the United States (respectively United Kingdom) commodities to \$US (respectively £Sterling), as well as connecting $\$$ US and £ Sterling. This symmetric relation is connected, $\bar{R}^{3}=N \times N$, and its graph is a tree so that $\# R=2(n-1)$.

In Example 8, the relation was a pair of monetary trade systems, each with a single money, joined together with an arc between the distinguished nodes $\$$ US and $£$ Sterling. This is a special case of a more general construction. The restrictions $E \subset \bar{R}^{n}$ and $\# R=2(n-1)$ are always satisfied by such trading post structures that are built by connecting smaller monetary trade systems, each with a single money: Begin with a distinguished point, $i_{0}$; Connect it to $n\left(i_{0}\right) \geq 1$ other points using a monetary trade system with money $i_{0}$; For each point $i_{1}$ in the resulting system, connect it to $n\left(i_{1}\right) \geq 0$ other points using a monetary trade system with money $i_{1}$. Such a process can be continued indefinitely, having $n\left(i_{t}\right)$ large gives a very "bushy" trading post structure with a small number of very heavily used moneys, having $n\left(i_{t}\right)$ small gives a "sprawling" trading post structure with many moneys.

Note that monetary trading structures arise without any distinctive designation of the properties of 'money' in the cost function; it is the cost structure of trade rather than a low cost of trading money that generates the concentration on common trading medium. Intuitively, it is clear that adding such complications to the cost structure would tend to concentrate the solution trading post structures on a smaller number of moneys. We investigate two factors that might lead to such concentration. The first is the existence of a "natural" money, the second is a formalization of Prof. Tobin's (1980) observation that the use of "a particular money by one individual increases its value to other actual or potential users." This last observation turns out to be intimately related (in our framework) to the rate of return dominance puzzle.

\subsection{A Natural Money}

As noted, the trading arrays that arise in Theorem 3 may have many moneys. Let $K$ be the set of 'moneys' in a tree, $K=\{k: \# R(k) \geq 2\}$. The cost of such an array is, ignoring marginal costs,

$$
\sum_{k \in K} \sum_{i \in R(k)} \alpha_{k i} .
$$


Now, a good $k$ is a natural money if most or all of the $\alpha_{k i}$ are relatively small. Thus, an immediate sufficient condition for $k$ to be a "natural" money is that the $\alpha_{k i}$ be small relative to the other $\alpha$ 's.

Corollary 9 Assume that $\widetilde{\beta} \simeq \mathbf{0}$. Suppose further that for some $k \in N$ and for all $i \neq k, \alpha_{k i} \simeq 0$ while $\alpha_{i j} \simeq \alpha$ for some $\alpha>0$ for all ij pairs not containing $k$. Then the unique solution to Symmetric $(n)$ is a monetary trade system with the single money $k$.

Proof: As indicated.

\subsection{Rate of Return Dominance}

One of the classic issues of microeconomic monetary theory is rate of return dominance, "What has to be explained is the decision to hold assets in the form of barren money, rather than of interest- or profit-yielding securities," Hicks (1935). The reply this model suggests is to ask "Are there solutions to $\operatorname{Symmetric}(m)$ that are dominated?" The answer to that question is "no." Symmetric $(m)$ asks for a global minimum, and a global minimum will be a minimum globally. Alternatively, is the choice of a monetary trading array self-enforcing locally even if it is dominated globally?

Prof. Tobin (1959) suggests that the answer is "yes,"

Why are some assets selected by a society as generally acceptable media of exchange while others are not? This is not an easy question because the answer is self-justifying .... The principal reason ... that Treasury bills are not media of exchange is that they are not generally acceptable. This unsatisfactory circular conclusion underlines the essential point, that general acceptability in exchange is one of those phenomena - like language, rules of the road, fashion in dress, - where the fact of social consensus is much more important and more predictable than the content.

Let us first consider incremental moves from one monetary trading array to another under the Conditions of Theorem 2, specifically, $m=2$, that all goods can be traded in two or fewer steps. For example, suppose that Treasury bills are a superior money in the sense of Corollary 4 but that society presently uses the single money, $\$$ US. Changing the trading post between good $i$ and $\$$ US and to a trading post between good $i$ and Treasury Bills lowers total costs, but it violates the condition 
that all goods be connected in two steps. The restriction $m=2$ in solving the problem Symmetric(2) secures the choice of a single monetary instrument against small perturbations in the trading array, even in the presence of a potentially superior monetary instrument.

This argument breaks down when $\mathrm{m}$ is unrestricted as in Theorem 3. Suppose also that $R$ is any monetary trading array that includes Treasury bills but for which Treasury bills are not the single money. Open any trading post between a good $i$ and Treasury bills and close any of the trading posts between $i$ and some other good $j$. This results in a lower cost connected trading array that may require more steps. Thus, provided $\mathrm{m}$ is unrestricted, small changes toward the best money in any alternative arrangement lead to improvements. Any money other than the best one is unstable to small changes in this sense.

This instability depends on there being no advantages to concentration per se. Suppose that instead of the $\alpha_{i j}$ 's being fixed independent of $R$ and the $v_{i j}^{h}$ 's, they depend on the volume of trade through the nodes. Specifically, suppose that the cost of trade through the good $k$ is and increasing and concave function of the total volume of trade through $k$. A natural money in this context would be one for which the concave function is uniformly lower than it is for any other good. With concavity, an inferior money may well prove to be stable to small changes. All that is needed is that the first small increases in trading volume with the superior money cost more than they cost when they were the last units traded with the inferior money.

Once the trading post array is monetary with a single money, there is a strong incentive for additional trading posts to use that money as well. There is a small scale economy built into the specification of the problem by the fixed cost $\alpha_{i j}$ (or the concave cost function) at each trading post. The interaction across posts through the implementation of the array of agents' excess demands turns this small scale economy into a large systemic scale economy. 


\section{References}

Clower, R. W. (1965), "The Keynesian Counterrevolution: A Theoretical Appraisal," in F. H.Hahn and F. P. R. Brechling, eds., The Theory of Interest Rates, London: Macmillan \& Co.

Granot, F. and A.F. Veinott, Jr. (1985), "Substitutes, Complements and Ripples in Network Flows," Mathematics of Operations Research, 10, 3, 471-497.

Haller, H. (1989a), "Topologies as Trade Infrastructures," Virginia Polytechnic Institute and State University, Department of Economics Working Paper, November.

Haller, H. (1989b), "Large Random Graphs in Pseudo-Metric Spaces," Virginia Polytechnic Institute and State University, Department of Economics Working Paper, November.

Hendricks, K., M. Piccione, and G. Tan (1992), "The Economics of Hubs: The Case of Monopoly," photocopy, University of British Columbia, Department of Economics.

Jevons, W. S. (1875), Money and the Mechanism of Exchange, London: H. S. King.

Lawler, Eugene (1976), Combinatorial Optimization, Networks and Matroids, New York: Holt, Rinehart, and Winston.

Ostroy, J. M. and R. M. Starr (1974), "Money and the Decentralization of Exchange," Econoometrica, 42, 1093-1113.

Ostroy, J. M. and R. M. Starr (1990), "The Transactions Role of Money," in Handbook of Monetary Economics, B. Friedman and F. H. Hahn, eds., Amsterdam: North Holland.

Rogawski, J. and M. Shubik (1986), "A Strategic Market Game with Transaction Costs," Mathhematical Social Sciences, 139-160.

Shubik, M. (1973), "Commodity Money, Oligopoly, Credit and Bankruptcy in a General Equiilibrium Model," Western Economic Journal, XI, March, 24-38.

Shubik, M. (1993), The Theory of Money and Financial Instititutions, Cambridge: MIT Press (forthcoming).

Starr, R. M. and M. B. Stinchcombe (1992), "Efficient Transportation Routing and Natural Monopoly in the Airline Industry: An Economic Analysis of Hub-Spoke and Related Sysstems," University of California, San Diego, Working Paper, May.

Stinchcombe, A.L. (1968), Constructing Social Theories. New York: Harcourt, Brace, and World.

Tobin, J. (1959), The Tobin Manuscript, New Haven: Yale University. Mimeographed.

Tobin, J. (1980), "Discussion," in Kareken, J. and N. Wallace, Models of Monetary Economies, Minneapolis: Federal Reserve Bank of Minneapolis. 
Walras, L. (1954), Elements of Pure Economics, Jaffe translation, Homewood, Illinois: Irwin. 